\title{
INDECOMPOSABLE MODULES AND GELFAND RINGS
}

\author{
FRANÇOIS COUCHOT
}

\begin{abstract}
It is proved that a commutative ring is clean if and only if it is Gelfand with a totally disconnected maximal spectrum. It is shown that each indecomposable module over a commutative ring $R$ satisfies a finite condition if and only if $R_{P}$ is an artinian valuation ring for each maximal prime ideal $P$. Commutative rings for which each indecomposable module has a local endomorphism ring are studied. These rings are clean and elementary divisor rings. It is shown that each commutative ring $R$ with a Hausdorff and totally disconnected maximal spectrum is local-global. Moreover, if $R$ is arithmetic then $R$ is an elementary divisor ring.
\end{abstract}

In this paper $R$ is a commutative ring with unity and modules are unitary.

In [11, Proposition 2] Goodearl and Warfield proved that each zero-dimensional $\operatorname{ring} R$ satisfies the second condition of our Theorem 1.1] and this condition plays a crucial role in their paper. In Section 1 we show that a ring $R$ enjoys this condition if and only if it is clean, if and only if it is Gelfand with a totally disconnected maximal spectrum. So we get a generalization of results obtained by Anderson and Camillo in [1] and by Samei in 21. We deduce that every commutative ring $R$ with a Hausdorff and totally disconnected maximum prime spectrum is local-global, and moreover, $R$ is an elementary divisor ring if, in addition, $R$ is arithmetic. One can see in 7 that local-global rings have very interesting properties.

In Section [3] we give a characterization of commutative rings for which each indecomposable module satisfies a finite condition: finitely generated, finitely cogenerated, cyclic, cocyclic, artinian, noetherian or of finite length. We deduce that a commutative ring is Von Neumann regular if and only if each indecomposable module is simple. This last result was already proved in [5]. We study commutative rings for which each indecomposable module has a local endomorphism ring. These rings are clean and elementary divisor rings. It remains to find valuation rings satisfying this property to give a complete characterization of these rings. We also give characterizations of Gelfand rings and clean rings by using properties of indecomposable modules. Similar results are obtained in Section 4 for commutative rings for which each prime ideal contains only one minimal prime ideal.

We denote respectively Spec $R$, Max $R$ and Min $R$, the space of prime ideals, maximal ideals, and minimal prime ideals of $R$, with the Zariski topology. If $A$ a subset of $R$, then we denote

$$
\begin{gathered}
V(A)=\{P \in \operatorname{Spec} R \mid A \subseteq P\}, D(A)=\{P \in \operatorname{Spec} R \mid A \not \subset P\}, \\
V_{M}(A)=V(A) \cap \operatorname{Max} R \text { and } D_{M}(A)=D(A) \cap \operatorname{Max} R .
\end{gathered}
$$

1991 Mathematics Subject Classification. Primary: 13C05; Secondary: 13 A99.

Key words and phrases. Gelfand ring, clean ring, indecomposable module, local-global ring, totally disconnected space, arithmetic ring. 


\section{LOCAL-GLOBAL GELFAND RINGS}

As in [19] we say that a commutative ring $R$ is Gelfand if each prime ideal is contained in only one maximal ideal. In this case, we put $\mu$ : Spec $R \rightarrow \operatorname{Max} R$ the map defined by $\mu(J)$ is the maximal ideal containing $J$ for each prime ideal $J$. Then $\mu$ is continuous and Max $R$ is Hausdorff by [17. Theorem 1.2].

In [1], Goodearl and Warfield proved that every zero-Krull-dimensional commutative ring satisfies the second condition of the following theorem. This property is used to show cancellation, $n$-root and isomorphic refinement theorems for finitely generated modules over algebras over a commutative ring which is Von Neumann regular modulo its Jacobson radical. So, the following theorem allows us to extend these results to each ring with a Hausdorff and totally disconnected maximal spectrum. As in 20] we say that a ring $R$ is clean if each element of $R$ is the sum of a unit with an idempotent. In [20, Proposition 1.8 and Theorem 2.1] Nicholson proved that commutative clean rings are exactly the exchange rings defined by Warfield in 24. In 21] Samei proved that the conditions (1), (3) and (4) are equivalent when $R$ is semiprimitive and in [1 Anderson and Camillo showed that each clean ring is Gelfand. We can also see [18, Theorem 3]. If $P$ is a prime ideal we denote by $0_{P}$ the kernel of the natural map $R \rightarrow R_{P}$.

Theorem 1.1. Let $R$ be a ring. The following conditions are equivalent:

(1) $R$ is a Gelfand ring and Max $R$ is totally disconnected.

(2) Each $R$-algebra $S$ satisfies this condition: let $f_{1}, \ldots, f_{k}$ be polynomials over $S$ in noncommuting variables $x_{1}, \ldots, x_{m}, y_{1}, \ldots, y_{n}$. Let $a_{1}, \ldots, a_{m} \in$ $S$. Assume that $\forall P \in \operatorname{Max} R$ there exists $b_{1}, \ldots, b_{n} \in S_{P}$ such that $f_{i}\left(a_{1}, \ldots, a_{m}, b_{1}, \ldots, b_{n}\right)=0 \forall i, 1 \leq i \leq k$. Then there exist $d_{1}, \ldots, d_{n} \in S$ such that $f_{i}\left(a_{1}, \ldots, a_{m}, d_{1}, \ldots, d_{n}\right)=0 \forall i, 1 \leq i \leq k$.

(3) $R$ is a clean ring.

(4) $R$ is Gelfand and $\forall P \in \operatorname{Max} R, 0_{P}$ is generated by a set of idempotents.

Proof. (1) $\Rightarrow(2)$. By [10, Theorem 16.17] Max $R$ has a base of clopen subsets. Since $\mu$ is continuous, each clopen subset of Max $R$ is of the form $D_{M}(e)$ for some idempotent $e$. So we can do the same proof as in [11 Proposition 2] where we replace Spec $R$ with Max $R$.

$(2) \Rightarrow(3)$. Let $a \in R$. We consider the following equations: $x^{2}=x$ and $y(a-x)=1$. Since each local ring is clean, these equations have a solution in $R_{P}$ for each maximal ideal $P$. We conclude that there is also a solution in $R$ and that $R$ is clean.

$(3) \Rightarrow(1)$. Let $P, P^{\prime} \in \operatorname{Max} R, P \neq P^{\prime}$. Then there exist $a \in P$ and $a^{\prime} \in P^{\prime}$ such that $a+a^{\prime}=1$. We have $a=u+e$ where $u$ is a unit and $e$ is an idempotent. Since $a \in P$ and $u \notin P$ we get that $e \notin P$. We have $a^{\prime}=1-a=-u+(1-e)$. So $1-e \notin P^{\prime}$. Consequently $P$ and $P^{\prime}$ have disjoint clopen neighbourhoods. Since Max $R$ is quasi-compact, we deduce that this space is compact and totally disconnected. The equality $e(1-e)=0$ implies that $P \cap P^{\prime}$ contains no prime ideal. Hence $R$ is Gelfand.

$(1) \Rightarrow(4)$. Let $P$ be a maximal ideal and $a \in 0_{P}$. Then there exists $s \in R \backslash P$ such that $s a=0$. Since $\operatorname{Max} R$ is totally disconnected there is a clopen subset $U$ such that $U \subseteq D_{M}(s)$. Because of $\mu$ is continuous, there exists an idempotent $e$ such that $P \in D(e)=\mu^{\leftarrow}(U) \subseteq \mu^{\leftarrow}\left(D_{M}(s)\right) \subseteq D(s)$. Then $e \in R s$, ea $=0$, $a=a(1-e)$ and $1-e \in 0_{P}$. 
(4) $\Rightarrow(1)$. Let $P, P^{\prime} \in \operatorname{Max} R, P \neq P^{\prime}$. Since $R$ is Gelfand, by 17, Theorem 1.2] there exist $a \in 0_{P} \backslash P^{\prime}$. Then there exists an idempotent $e \in 0_{P} \backslash P^{\prime}$. Clearly $1-e \notin P$. Consequently $P$ and $P^{\prime}$ have disjoint clopen neighbourhoods.

We say that $R$ is local-global if each polynomial over $R$ in finitely many indeterminates which admits unit values locally, admits unit values. Recall that most of the results of [1] about commutative rings which are Von Neumann regular modulo their Jacobson radicals, have been extended to local-global rings by Estes and Guralnick in [7. We have the following corollary:

Corollary 1.2. Let $R$ be a ring such that Max $R$ is Hausdorff and totally disconnected. Then $R$ is local-global.

Proof. Let $J$ be the Jacobson radical of $R$. Then $R$ is local-global if and only if $R / J$ is local-global. So we may assume that $R$ is semiprimitive. From the remark that follows [17. Theorem 1.2] and from Theorem [1.1] we deduce that $R$ is clean. Let $f$ be a polynomial over $R$ in finitely many indeterminates $X_{1}, \ldots, X_{n}$, which admits unit values locally. Then, we apply theorem 1.1 by taking $S=R$ to the polynomial $Y f\left(X_{1}, \ldots, X_{n}\right)-1$.

Remark 1.3. If $R$ is the ring of algebraic integers, then $R$ is local-global by [ 6 ] and semi-primitive. But this ring is not Gelfand.

\section{Arithmetic Gelfand Rings}

We say that a module is uniserial if its set of submodules is totally ordered by inclusion, we say that a ring $R$ is a valuation ring if it is uniserial as $R$-module and we say that $R$ is arithmetic if $R_{P}$ is a valuation ring for each maximal ideal $P$. Recall that $R$ is a Bézout ring if each finitely generated ideal is principal and $R$ is an elementary divisor ring if each finitely presented module is a direct sum of cyclic submodules.

Theorem 2.1. Let $R$ be an arithmetic local-global ring. Then $R$ is an elementary divisor ring. Moreover, for each $a, b \in R$, there exist $d, a^{\prime}, b^{\prime}, c \in R$ such that $a=a^{\prime} d, b=b^{\prime} d$ and $a^{\prime}+c b^{\prime}$ is a unit of $R$.

Proof. Since every finitely generated ideal is locally principal $R$ is Bézout by $[7$ Corollary 2.7]. Let $a, b \in R$. Then there exist $a^{\prime}, b^{\prime}, d \in R$ such that $a=a^{\prime} d, b=b^{\prime} d$ and $R a+R b=R d$. Consider the following polynomial $a^{\prime}+b^{\prime} T$. If $P$ is a maximal ideal, then we have $a R_{P}=d R_{P}$ or $b R_{P}=d R_{P}$. So, either $a^{\prime}$ is a unit of $R_{P}$ and $a^{\prime}+b^{\prime} r$ is a unit of $R_{P}$ for each $r \in P R_{P}$, or $b^{\prime}$ is a unit of $R_{P}$ and $a^{\prime}+b^{\prime}\left(1-a^{\prime} / b^{\prime}\right)$ is a unit of $R_{P}$. We conclude that the last assertion holds. Now, let $a, b, c \in R$ such that $R a+R b+R c=R$. We set $R b+R c=R d$. Let $b^{\prime}, c^{\prime}, s$ and $q$ such that $b=b^{\prime} d, c=c^{\prime} d$ and $b^{\prime}+c^{\prime} q$ and $a+s d$ are units. Then $\left(b^{\prime}+c^{\prime} q\right)(a+s d)=\left(b^{\prime}+c^{\prime} q\right) a+s(b+q c)$ is a unit. We conclude by [ $[$ Theorem 6].

We deduce the following corollary which is a generalization of [4, Theorem III.6] and [9, Theorem 5.5].

Corollary 2.2. Let $R$ be an arithmetic ring with a Hausdorff and totally disconnected maximal spectrum. Then $R$ is an elementary divisor ring. Moreover, for each $a, b \in R$, there exist $d, a^{\prime}, b^{\prime}, c \in R$ such that $a=a^{\prime} d, b=b^{\prime} d$ and $a^{\prime}+c b^{\prime}$ is a unit of $R$. 
Corollary 2.3. Let $R$ be an arithmetic Gelfand ring such that Min $R$ is compact. Then $R$ is an elementary divisor ring. Moreover, for each $a, b \in R$, there exist $d, a^{\prime}, b^{\prime}, c \in R$ such that $a=a^{\prime} d, b=b^{\prime} d$ and $a^{\prime}+c b^{\prime}$ is a unit of $R$.

Proof Let $\mu^{\prime}$ be the restriction of $\mu$ to Min $R$. Since $R$ is arithmetic each prime ideal contains only one minimal prime ideal. Then $\mu^{\prime}$ is bijective and it is an homeomorphism because Min $R$ is compact. One can apply corollary 2.2 since Min $R$ is totally disconnected .

Remark 2.4. In 9 there is an example of a Gelfand Bézout ring $R$ which is not an elementary divisor ring. Consequently Min $R$ is not compact.

\section{INDECOMPosable MOdULES AND MAXIMAL IDEALS}

In the two next propositions we give a characterization of Gelfand rings and clean rings by using properties of indecomposable modules.

Proposition 3.1. Let $R$ be a ring. The following conditions are equivalent:

(1) For each $R$-algebra $S$ and for each left $S$-module $M$ for which $\operatorname{End}_{S}(M)$ is local, Supp $M$ contains only one maximal ideal.

(2) $R$ is a Gelfand ring.

(3) $\forall P \in \operatorname{Max} R$ the natural map $R \rightarrow R_{P}$ is surjective.

When these conditions are satisfied, $M=M_{P}$ for each left $S$-module $M$ for which $\operatorname{End}_{S}(M)$ is local, where $P$ is the unique maximal ideal of Supp $M$ and where $S$ is an algebra over $R$.

Proof. Assume that $R$ is Gelfand. Let $S$ be an $R$-algebra and let $M$ be a left $S$-module such that $\operatorname{End}_{S}(M)$ is local. Let $P$ be the prime ideal which is the inverse image of the maximal ideal of $\operatorname{End}_{S}(M)$ by the canonical map $R \rightarrow \operatorname{End}_{S}(M)$ and let $Q=\mu(P)$. Since $M$ is an $R_{P}$-module, $0_{Q} \subseteq \operatorname{ann}_{R}(M)$. So, Supp $M \subseteq V\left(0_{Q}\right)$ and $Q$ is the only maximal ideal belonging to $V\left(0_{Q}\right)$ since $R$ is Gelfand.

Conversely, if $P$ is a prime ideal then $R_{P}=\operatorname{End}_{R}\left(R_{P}\right)$. It follows that $P$ is contained in only one maximal ideal.

By [17. Theorem 1.2] $R$ is Gelfand if and only if, $\forall P \in \operatorname{Max} R, P$ is the only maximal ideal containing $0_{P}$. This is equivalent to $R / 0_{P}$ is local, $\forall P \in \operatorname{Max} R$. It is obvious that $R_{P}=R / 0_{P}$ if $R / 0_{P}$ is local. (When $R$ is semi-primitive we can see [3. Proposition 1.6.1]).

Recall that the diagonal map $M \rightarrow \Pi_{P^{\prime} \in \operatorname{Max}(R)} M_{P^{\prime}}$ is monic. Since $R$ is Gelfand, we have $M_{P}=M / 0_{P} M$ where $P$ is the only maximal ideal of Supp $M$. Hence $M=M_{P}$.

Proposition 3.2. Let $R$ be a ring. The following conditions are equivalent:

(1) For each $R$-algebra $S$ and for each indecomposable left $S$-module $M$, Supp $M$ contains only one maximal ideal.

(2) $R$ is clean.

When these conditions are satisfied, $M=M_{P}$ for each indecomposable left $S$-module $M$, where $P$ is the unique maximal ideal of Supp $M$ and $S$ is an $R$-algebra.

Proof. $(2) \Rightarrow(1)$. By Theorem 1.1 Max $R$ is totally disconnected. So, if $P$ and $P^{\prime}$ are two different maximal ideals such that $P \in \operatorname{Supp} M$ then there exists an idempotent $e \in P \backslash P^{\prime}$ because $\mu$ is continuous. Since $(1-e) \notin P$ and $M_{P} \neq 0$, we have $(1-e) M \neq 0$. We deduce that $e M=0$ and $M_{P^{\prime}}=0$. 
$(1) \Rightarrow(2) . R$ is Gelfand by Proposition 3.1 Let $A$ be an ideal such that $V(A)$ is the inverse image of a connected component of Max $R$ by $\mu$. Then $V(A)$ is connected too, whence $R / A$ is indecomposable. So there is only one maximal ideal in $V(A)$. Since each connected component contains only one point, Max $R$ is totally disconnected.

This lemma is needed to prove the main results of this section.

Lemma 3.3. Let $R$ be a local ring which is not a valuation ring. Then there exists an indecomposable non-finitely generated $R$-module whose endomorphism ring is not local.

Proof. Since $R$ is not a valuation ring there exist $a, b \in R$ such that neither divides the other. By taking a suitable quotient ring, we may assume that $R a \cap R b=$ 0 and $P a=P b=0$. Let $F$ be a free module generated by $\left\{e_{n} \mid n \in \mathbb{N}\right\}$, let $K$ be the submodule generated by $\left\{a e_{n}-b e_{n+1} \mid n \in \mathbb{N}\right\}$ and let $M=F / K$. Clearly $M / P M \cong F / P F$. We will show that $M$ is indecomposable and $S:=\operatorname{End}_{R}(M)$ is not local. Let us observe that $M$ is defined as in proof of [12, Theorem 2.3]. But, since $R$ is not necessarily artinian, we do a different proof to show that $M$ is indecomposable. We shall prove that $S$ contains no trivial idempotents. Let $s \in S$. Then $s$ is induced by an endomorphism $\tilde{s}$ of $F$ which satisfies $\tilde{s}(K) \subseteq K$. For each $n \in \mathbb{N}$ there exists a finite family $\left(\alpha_{p, n}\right)$ of elements of $R$ such that:

$$
\tilde{s}\left(e_{n}\right)=\sum_{p \in \mathbb{N}} \alpha_{p, n} e_{p}
$$

Since $\tilde{s}(K) \subseteq K, \forall n \in \mathbb{N}, \exists$ a finite family $\left(\beta_{p, n}\right)$ of elements of $R$ such that:

$$
\tilde{s}\left(a e_{n}-b e_{n+1}\right)=\sum_{p \in \mathbb{N}} \beta_{p, n}\left(a e_{p}-b e_{p+1}\right)
$$

From [1] and [2 if follows that:

$$
\sum_{p \in \mathbb{N}}\left(a \alpha_{p, n}-b \alpha_{p, n+1}\right) e_{p}=a \beta_{0, n} e_{0}+\sum_{p \in \mathbb{N}^{*}}\left(a \beta_{p, n}-b \beta_{p-1, n}\right) e_{p}
$$

Since $P a=P b=R a \cap R b=0$ we deduce that

$$
\alpha_{0, n+1} \equiv 0[P], \quad \alpha_{p, n} \equiv \beta_{p, n}[P] \text { and } \alpha_{p, n+1} \equiv \beta_{p-1, n}[P]
$$

It follows that

$$
\text { (i) } \alpha_{p, n} \equiv \alpha_{p+1, n+1}[P], \forall p, n \in \mathbb{N} \text {, and (ii) } \alpha_{p, p+k+1} \equiv 0[P], \forall p, k \in \mathbb{N}
$$

Now we assume that $s$ is idempotent. Let $x_{n}=e_{n}+K, \forall n \in \mathbb{N}$. Let $\bar{s}$ be the endomorphism of $M / P M$ induced by $s$. If $L$ is an $R$-module and $x$ an element of $L$, we put $\bar{x}=x+P L$. From $s^{2}\left(x_{0}\right)=s\left(x_{0}\right)$ we get the following equality:

$$
\sum_{n \in \mathbb{N}}\left(\sum_{p \in \mathbb{N}} \alpha_{n, p} \alpha_{p, 0}\right) x_{n}=\sum_{n \in \mathbb{N}} \alpha_{n, 0} x_{n}
$$

Then $\bar{\alpha}_{0,0}=\sum_{p \in \mathbb{N}} \bar{\alpha}_{0, p} \bar{\alpha}_{p, 0}=\bar{\alpha}_{0,0}^{2}$, since $\bar{\alpha}_{0, p}=0$ by $3(i i), \forall p>0$. So, we have $\bar{\alpha}_{0,0}=0$ or $\bar{\alpha}_{0,0}=1$. If $\bar{\alpha}_{0,0}=1$ then we replace $s$ with $\mathbf{1}_{M}-s$. So we may assume that $\bar{\alpha}_{0,0}=0$. By $3(i) \bar{\alpha}_{n, n}=0, \forall n \in \mathbb{N}$. By using 4 and $\mathbb{B}(i i)$ we get that

$$
\bar{\alpha}_{n, 0}=\sum_{p=0}^{n-1} \bar{\alpha}_{n, p} \bar{\alpha}_{p, 0}+\bar{\alpha}_{n, n} \bar{\alpha}_{n, 0}
$$


Hence, if $\bar{\alpha}_{p, 0}=0, \forall p<n$ then $\bar{\alpha}_{n, 0}=0$ too. By induction we obtain that $\bar{\alpha}_{n, 0}=0$, $\forall n \in \mathbb{N}$. We deduce that

$$
\alpha_{p, n} \in P, \forall p, n \in \mathbb{N}
$$

Let $A=\operatorname{Im} s, B=\operatorname{Ker} s$ and let $A^{\prime}$ and $B^{\prime}$ be the inverse image of $A$ and $B$ by the natural map $F \rightarrow M$. If $x \in A^{\prime}$ then $\tilde{s}(x)=x+y$ for some $y \in K$. By 5 and $P a=P b=0$ it follows that $\tilde{s}(y)=0$ and $\tilde{s}^{2}(x)=\tilde{s}(x)$. If $x \in B^{\prime}$ then $\tilde{s}(x) \in K$. So $\tilde{s}^{2}(x)=0$. We deduce that $\left(\tilde{s}^{2}\right)^{2}=\tilde{s}^{2}$. Let $C=\operatorname{Im} \tilde{s}^{2}$. Then $C$ is projective and $C=P C$ by [5 By [2, Proposition 2.7] $C=0$. So $s=0$ (or $\mathbf{1}_{M}-s=0$ ).

It remains to prove that $S$ is not local. Let $f, g \in S$ defined in the following way: $f\left(x_{n}\right)=x_{n+1}$ and $g\left(x_{n}\right)=x_{n}-x_{n+1}, \forall n \in \mathbb{N}$. We easily check that $x_{0} \notin \operatorname{Im} f \cup \operatorname{Im} g$. So $f$ and $g$ are not units of $S$ and $f+g=\mathbf{1}_{M}$ is a unit. Hence $S$ is not local.

A module is cocyclic (respectively finitely cogenerated) if it is a submodule of the injective hull of a simple module (respectively of a finite direct sum of injective hulls of simple modules).

Now we give a characterization of commutative rings for which each indecomposable module satisfies a finite condition.

Theorem 3.4. Let $R$ be a ring. The following conditions are equivalent:

(1) Each indecomposable $R$-module is of finite length.

(2) Each indecomposable $R$-module is noetherian.

(3) Each indecomposable $R$-module is finitely generated.

(4) Each indecomposable $R$-module is artinian.

(5) Each indecomposable R-module is finitely cogenerated.

(6) Each indecomposable $R$-module is cyclic.

(7) Each indecomposable $R$-module is cocyclic.

(8) For each maximal ideal $P, R_{P}$ is an artinian valuation ring.

(9) $R$ is an arithmetic ring of Krull-dimension 0 and its Jacobson ideal $J$ is T-nilpotent.

Proof. The following implications are obvious: (1) $\Rightarrow(2) \Rightarrow(3),(1) \Rightarrow(4) \Rightarrow$ $(5),(6) \Rightarrow(3)$ and $(7) \Rightarrow(5)$.

$(8) \Rightarrow(1),(6)$ and $(7)$. $R$ has Krull dimension 0 . Hence $R$ is clean. So, if $M$ is an indecomposable module, by proposition 3.2 there is only one maximal ideal $P$ such that $M_{P} \neq 0$. Moreover $M \cong M_{P}$. We conclude by [12 Theorem 4.3].

$(3) \Rightarrow(8)$. Let $P$ be a maximal ideal and $E$ the injective hull of $R / P$. Then each submodule of $E$ is indecomposable. It follows that $E$ is a noetherian module. By [23. Proposition 3] $E$ is a module of finite length, and by [23. Theorem 3] $R_{P}$ is artinian. We conclude by 12, Theorem 2.3] or Lemma 3.3.

$(5) \Rightarrow(8)$. Let $P$ be a maximal ideal. Then each factor of $R_{P}$ modulo an ideal of $R_{P}$ is finitely cogenerated. It follows that $R_{P}$ is artinian. We conclude as above.

$(8) \Rightarrow(9)$. Let $x_{1}, \ldots, x_{n}, \ldots$ be a sequence of elements of $J$. Then for each maximal ideal $P \exists s_{P} \notin P$ and a positive integer $n_{P}$ such that $s_{P} x_{1} \ldots x_{n_{P}}=0$. There is a finite family of open sets $D\left(s_{n_{P_{1}}}\right), \ldots, D\left(s_{n_{P_{m}}}\right)$ that cover Spec $R$. We set $n=\max \left\{n_{P_{1}}, \ldots, n_{P_{m}}\right\}$. Then $x_{1} \ldots x_{n}=0$.

(9) $\Rightarrow(8) . \forall P \in \operatorname{Max} R, R_{P}$ is a valuation ring and $P R_{P}$ is a nilideal. Then for every $r \in P$ there exists $s \in R \backslash P$ such that $s r$ is nilpotent. So we get that $P R_{P}=J R_{P}$, whence $P R_{P}$ is T-nilpotent. We easily prove that $R_{P}$ is artinian. 
From this theorem it is easy to deduce the two following corollaries. Another proof of the second corollary is given in [5. Theorem 2.13].

Corollary 3.5. Let $n$ be a positive integer, $R$ a ring and $J$ its Jacobson radical. Then the following conditions are equivalent:

(1) Each indecomposable module has a length $\leq n$.

(2) For each maximal ideal $P, R_{P}$ is a valuation ring and $\left(P R_{P}\right)^{n}=0$.

(3) $R$ is an arithmetic ring of Krull-dimension 0 and $J^{n}=0$.

Corollary 3.6. A ring $R$ is Von Neumann regular if and only if every indecomposable module is simple.

The next theorem gives a partial characterization of commutative rings for which each indecomposable module has a local endomorphism ring.

Theorem 3.7. Let $R$ be a ring for which $\operatorname{End}_{R}(M)$ is local for each indecomposable module $M$. Then $R$ is a clean elementary divisor ring.

Proof. Let $P$ be a prime ideal. Then $R / P=\operatorname{End}_{R}(R / P)$ is local. Hence $R$ is Gelfand. We prove that Max $R$ is totally disconnected as in proof of proposition 3.2 If $P$ is a maximal ideal, each indecomposable $R_{P}$-module $M$ is also indecomposable over $R$ and $\operatorname{End}_{R}(M)=\operatorname{End}_{R_{P}}(M)$. By Lemma $3.3 R_{P}$ is a valuation ring.

Example 3.8. If $R$ is a ring satisfying the equivalent conditions of Theorem 3.4 then each indecomposable $R$-module has a local endomorphism ring. But, by [14, Corollary 2 p.52] and [22, Corollary 3.4], each complete discrete rank one valuation ring enjoys this property too. So, we consider a complete discrete rank one valuation ring $D, Q$ its ring of fractions and $R$ the subring of $Q^{\mathbb{N}}$ defined as in 20, Example 1.7]: $x=\left(x_{n}\right)_{n \in \mathbb{N}} \in R$ if $\exists p \in \mathbb{N}$ and $s \in D$ such that $x_{n}=s, \forall n>p$. Since $D$ is local, $R$ is clean and semi-primitive by [18, Theorem 2]. We put $\mathbf{1}=\left(\delta_{n, n}\right)_{n \in \mathbb{N}}$ and $\forall p \in \mathbb{N}, \mathbf{e}_{p}=\left(\delta_{p, n}\right)_{n \in \mathbb{N}}$ where $\delta_{n, p}$ is the Kronecker symbol. Let $J$ be the maximal ideal of $D$. If $P$ is a maximal ideal of $R$, then either $\mathbf{e}_{p} \in P, \forall p \in \mathbb{N}$, whence $P=J \mathbf{1}+\oplus_{p \in \mathbb{N}} R \mathbf{e}_{p}$ and $R_{P} \cong R / \oplus_{p \in \mathbb{N}} R \mathbf{e}_{p} \cong D$, or $\exists p \in \mathbb{N}$ such that $\mathbf{e}_{p} \notin P$, whence $P=R\left(\mathbf{1}-\mathbf{e}_{p}\right)$ and $R_{P} \cong R / P \cong Q$. Thus $R$ is arithmetic and each indecomposable $R$-module has a local endormorphism ring. Observe that each indecomposable $R$-module is uniseriel and linearly compact and its endomorphism ring is commutative.

\section{INDECOMPOSABLE MODULES AND MINIMAL PRIME IDEALS}

In this section we study rings $R$ for which each prime ideal contains only one minimal prime ideal. In this case, if $P \in$ Spec $R$, let $\lambda(P)$ be the only minimal prime ideal contained in $P$. We shall see that $\lambda$ is continuous if and only if Min $R$ is compact. (See [15, Theorem 2] when $R$ is semi-prime). But, since $\lambda$ is surjective, the set of minimal primes can be endowed with the quotient topology induced by the Zariski topology of Spec $R$. We denote this topologic space by QMin $R$. Then we have the following:

Proposition 4.1. Let $R$ be a ring such that each prime ideal contains a unique minimal prime ideal and $N$ its nilradical. Then QMin $R$ is compact. Moreover, QMin $R$ and Min $R$ are homeomorphic if and only if Min $R$ is compact.

The following lemma is needed to prove this proposition. This lemma is a generalization of [13 Lemma 2.8]. We do a similar proof. 
Lemma 4.2. Let $R$ be a ring, $N$ its nilradical and $a \in R \backslash N$. Let $P$ be a prime ideal such that $P /(N: a)$ is minimal in $R /(N: a)$. Then $P$ is a minimal prime ideal.

Proof. First we show that $a+(N: a)$ is a non-zerodivisor in $R /(N: a)$ and consequently $a \notin P$. Let $b \in R$ such that $a b \in(N: a)$. Then $a^{2} b \in N$. We easily deduce that $a b \in N$, whence $b \in(N: a)$. Let $r \in P$. Then there exist a positive integer $n$ and $s \in R \backslash P$ such that $s r^{n} \in(N: a)$. It follows that $a s r^{n} \in N$. Since as $\notin P$ we deduce that $P R_{P}$ is a nilideal, whence $P$ is a minimal prime.

Proof of proposition 4.1. Let $A$ and $B$ be two distinct minimal prime ideals. Since each maximal ideal contains only one minimal prime ideal, we have $A+B=R$. Therefore there exist $a \in A$ and $b \in B$ such that $a+b=1$. Thus $a \notin B$ and $a \notin N$. But $a$ is a nilpotent element of $R_{A}$. Hence $(N: a) \nsubseteq A$. In the same way we show that $B \in D((N: b))$. We have $(N: a) \cap(N: b)=(N: R a+R b)=N$. So $D((N: a)) \cap D((N: b))=\emptyset$. By Lemma 4.2 $D((N: a))$ and $D((N: b))$ are the inverse images of disjoint open subsets of QMin $R$ by $\lambda$. We conclude that this space is Hausdorff. Since Spec $R$ is quasi-compact, it follows that QMin $R$ is compact.

Let $\lambda^{\prime}$ be the restriction of $\lambda$ to $\operatorname{Min} R$. It is obvious that $\left(\lambda^{\prime}\right)^{-1}$ is continuous if and only if Min $R$ is compact.

Remark 4.3. If we consider the set of D-components of Spec $R$, defined in 16, endowed with the quotient topology, we get a topologic space $X$. Then $X$ is homeomorphic to Max $R$ (respectively QMin $R$ ) if $R$ is Gelfand (respectively every prime ideal contains only one minimal prime). But $X$ is not generally Hausdorff: see [16, Propositions 6.2 and 6.3].

Now we can show the two following propositions which are similar to Propositions 3.1 and 3.2 The proofs are similar too.

Proposition 4.4. Let $R$ be a ring. The following conditions are equivalent:

(1) For each $R$-algebra $S$ and for each left $S$-module $M$ for which $\operatorname{End}_{S}(M)$ is local, there exists only one minimal prime ideal A such that Supp $M \subseteq$ $V(A)$.

(2) Every prime ideal contains only one minimal prime ideal.

Proof. (2) $\Rightarrow(1)$. Let $S$ be an $R$-algebra and let $M$ be a left $S$-module such that $\operatorname{End}_{S}(M)$ is local. Let $P$ be the prime ideal which is the inverse image of the maximal ideal of $\operatorname{End}_{S}(M)$ by the canonical map $R \rightarrow \operatorname{End}_{S}(M), A=\lambda(P)$ and $0_{P}$ the kernel of the natural map $R \rightarrow R_{P}$. Since $M$ is an $R_{P}$-module, $0_{P} \subseteq \operatorname{ann}_{R}(M)$. It is obvious that $0_{P} \subseteq A$. On the other hand, $A R_{P}$ is the nilradical of $R_{P}$. It follows that $\operatorname{rad}\left(0_{P}\right)=A$. Hence we get that Supp $M \subseteq V\left(\operatorname{ann}_{R}(M)\right) \subseteq V\left(0_{P}\right)=V(A)$. If $B$ is another minimal prime, it is obvious that $V(A) \cap V(B)=\emptyset$.

$(1) \Rightarrow(2)$. If $P$ is a prime ideal then $R_{P}=\operatorname{End}_{R}\left(R_{P}\right)$. It follows that $P$ contains only one minimal prime ideal.

Proposition 4.5. Let $R$ be a ring. The following conditions are equivalent:

(1) For each $R$-algebra $S$ and for each indecomposable left $S$-module $M$, there is only one minimal prime ideal $A$ such that Supp $M \subseteq V(A)$. 
(2) Each prime ideal contains a unique minimal prime ideal and QMin $R$ is totally disconnected.

Proof. $\quad(1) \Rightarrow(2)$. By proposition 4.4 each prime ideal contains a unique minimal prime ideal. Let $P \in$ QMin $R$ and $C$ its connected component. There exists an ideal $A$ such that $V(A)=\lambda^{\leftarrow}(C)$. Then $V(A)$ is connected. It follows that $R / A$ is indecomposable. So $V(A)=V(P)$ and $C=\{P\}$.

$(2) \Rightarrow(1)$. Let $S$ be an $R$-algebra and $M$ be an indecomposable left $S$-module. Let $P \in \operatorname{Supp} M, A=\lambda(P), P^{\prime} \in \operatorname{Spec} R \backslash V(A)$ and $A^{\prime}=\lambda\left(P^{\prime}\right)$. Since QMin $R$ is totally disconnected, there exists an idempotent $e \in A \backslash A^{\prime}$. We easily deduce that $e \in P \backslash P^{\prime}$. Now we do as in the proof of Proposition 3.2 to conclude.

\section{REFERENCES}

[1] D. D. Anderson; V. Camillo, Commutative rings whose elements are a sum of a unit and an idempotent. Comm. Algebra 30(7) (2002), 3327-3336.

[2] H. Bass, Finistic dimension and a homological generalization of semi-primary rings. Trans. Amer. Math. Soc. 95 (1960), 466-488.

[3] R. Bkouche, Couples spectraux et faisceaux assocés. Applications aux anneaux de fonctions. Bull. Soc. Math. Fr, 98, 1970, 253-295.

[4] F. Couchot, The $\lambda$-dimension of commutative arithmetic rings, Comm. Algebra, 31(7), (2003), 3143-3158.

[5] F. Couchot, Modules with RD-composition series over a commutative ring, Comm. Algebra, 31(7), (2003), 3171-3194.

[6] E. C. Dade; Algebraic integral representations by arbitrary forms, Mathematika 10, 96-100, 1963; A correction, Mathematika 11, 89-90, 1964.

[7] D. R. Estes and R. M. Guralnick, Module equivalences: Local to global. When primitive polynomials represent units. J. Algebra 77, 138-157 (1982).

[8] L. Gillman and M. Henriksen, Some remarks about elementary divisor rings. Trans. Amer. Math. Soc. 82, 1956, 362-365.

[9] L. Gillman and M.Henriksen, Rings of continuous functions in which every finitely ideal is principal. Trans. Amer. Math. Soc. 82, 1956, 366-391.

[10] L. Gillman and M. Jerison, Rings of continuous functions, University Series in Higher Math., Van Nostrand, Princeton, N. J., 1960.

[11] K. R. Goodearl and R. B. Warfield, Algebras over Zero-Dimensional Rings, Math. Ann. 223, 1976, 157-168.

[12] P. Griffith, On the Decomposition of Modules and Generalized Left Uniserial Rings. Math. Ann. 184 (1970), 300-308.

[13] M. Henriksen and M. Jerison, The space of minimal prime ideals of a commutative ring. Trans. Amer. Math. Soc. 115, 1965, 110-130.

[14] I. Kaplansky, Infinite Abelian Groups. Ann Arbor, the University of Michigan Press (1969).

[15] J. Kist, Two characterizations of commutative Baer rings, Pac. J. Math. 50(1), 1974, 125-134.

[16] Lazard, D. Disconnexités des spectres d'anneaux et des préschémas. Bull. Soc. Math. Fr. 95, 95-108 (1967).

[17] G. De Marco and A. Orsati, Commutative rings in which every prime ideal is contained in a unique maximal ideal. Proc. Amer. Math. Soc. 30(3) (1971), 459-466.

[18] G. S. Monk, A characterization of exchange rings, Proc. Amer. Math. Soc. 35(2) (1972), 349-353.

[19] C. J. Mulvey, A generalization of Gelfand duality. J. Algebra, 56, 499-505, 1979.

[20] W. K. Nicholson, Lifting idempotents and exchange rings. Trans. Amer. Path. Soc. 229 (1977), 269-278.

[21] K. Samei, Clean elements in commutative reduced rings. Comm. Algebra, 32(9) (2004), 3479-3486.

[22] T.S. Shores and W.J. Lewis, Serial modules and endomorphism rings. Duke Math. J. 41, (1974), 889-909.

[23] P. Vámos, The dual of the notion of "finitely generated". J. London Math. Soc., 43 (1968), 643-646. 
[24] R. B. Warfield, Exchange rings and decompositions of modules. Math. Ann., 199 (1972), $31-36$.

Laboratoire de Mathématiques Nicolas Oresme, CNRS UMR 6139, Département de mathématiques et méCanique, 14032 Caen Cedex, France

E-mail address: couchot@math.unicaen.fr 